\title{
Chapitre 11
}

\section{Aperçus sur quelques logiciels de simulation utilisés pour des études en support à la conception et aux analyses de sûreté des réacteurs de recherche français}

La conception ou les modifications des réacteurs de recherche ou de leurs dispositifs expérimentaux (y compris les modifications des cœurs), de même que la démonstration de leur sûreté - y compris lors de réévaluations de sûreté -, s'appuient sur des études réalisées le plus souvent avec des logiciels de simulation ${ }^{320}$ dans divers domaines : neutronique ou criticité (cœurs et zones dédiées à l'entreposage de combustibles), thermohydraulique (cœurs, circuits de refroidissement), mécanique des structures (structures métalliques, ouvrages de génie civil)... Au premier chef, ce sont les exploitants (CEA, Institut Laue-Langevin) qui réalisent de telles études, mais l'IRSN peut également être amené à en réaliser lors de son expertise des dossiers transmis par ces exploitants à l’Autorité de sûreté nucléaire.

La validation d'un logiciel de simulation est bien entendu un aspect important en amont de son utilisation pour les études. Aussi, dans le cadre d'une démonstration de sûreté - ou de l'expertise d'une telle démonstration -, la capacité de chaque logiciel de

320. L'expression « code de calcul » est aussi utilisée. 
simulation à représenter correctement ou de manière conservative les phénomènes physiques mis en jeu doit être préalablement établie.

À cet égard, le paragraphe 8.3 relatif à l'accident de référence dit BORAX présente des exemples d'essais globaux à caractère démonstratif réalisés dans des réacteurs ou sur des maquettes pour conforter certaines évaluations faites par le calcul. Ce type de vérification peut en effet être souhaitable, voire indispensable, dans les cas où les évaluations faites par le calcul sont sujettes ou aboutissent à des incertitudes trop importantes (y compris du fait de simplifications de modélisation) ou lorsque les logiciels n'ont fait l'objet que d'une validation séparée de leurs différents modèles physiques.

Il convient aussi de rappeler ici l'importance toute particulière, pour un nouveau réacteur (ou pour un réacteur ayant fait l'objet de modifications substantielles), des essais de démarrage (ou de redémarrage), réalisés par l'exploitant sur différents matériels ou systèmes pour s'assurer, autant que cela est possible ${ }^{321}$, qu'ils sont aptes à remplir les missions pour lesquelles ils ont été conçus, avec les performances attendues issues des études de conception fondées en grande partie sur l'utilisation de logiciels de simulation.

Quelques-uns des logiciels utilisés ${ }^{322}$, dans des versions améliorées au fil du temps, et de leurs utilisations les plus notables ${ }^{323}$ pour les réacteurs de recherche français sont décrits succinctement ci-après. Cette description se limite pour l'essentiel aux possibilités offertes par ces logiciels, au contexte et aux buts des études pour lesquelles ils sont utilisés, ainsi qu'à certains aspects concernant les modélisations et les précautions à prendre pour obtenir une confiance satisfaisante dans les résultats obtenus - recalages sur des expériences ou comparaison de différents logiciels.

Un certain nombre de ces logiciels ont été initialement développés pour les réacteurs de puissance (par exemple FLICA, CATHARE, SIMMER). Des adaptations ont été réalisées pour leur utilisation pour des réacteurs de recherche (en particulier pour les réacteurs utilisant des combustibles à base d'uranium et d'aluminium, sous formes de plaques, avec de l'eau lourde comme fluide caloporteur ou modérateur...). Par ailleurs, des couplages réalisés entre logiciels de neutronique et de thermohydraulique de cœurs et de circuits de réacteurs peuvent être utilisés ${ }^{324}$, tels que CRONOS-FLICA, CRONOS-CATHARE ou encore CRONOS-FLICA-CATHARE - ce dernier couplage constituant la chaîne HEMERA (Highly Evolutionary Methods for Extensive Reactor Analysis ${ }^{325}$ ).

Il peut enfin être souligné que la complexité des cœurs de réacteurs de recherche, associant éléments combustibles standards, éléments combustibles pouvant contenir en partie des absorbants neutroniques, éléments absorbants en cœur ou à sa proximité, dispositifs expérimentaux très divers dans différents emplacements du cœur ou de sa

321. En effet, il n'est pas envisageable de provoquer des situations accidentelles pour s'assurer du bon fonctionnement des équipements ayant pour rôle de les maîtriser.

322. Voir, notamment, l'ouvrage "La neutronique », monographie de la Direction de l'énergie nucléaire du CEA, édition Le Moniteur, 2013.

323. Les utilisations dont il est fait état dans ce chapitre ont fait l'objet de publications.

324. Bien que réalisés pour les réacteurs à eau sous pression, certains de ces couplages ont été ou peuvent être utilisés pour certains réacteurs de recherche, d'autres nécessitant des adaptations.

325. Méthodes hautement évolutives pour une analyse approfondie des réacteurs. 
périphérie (par exemple des boucles pouvant être refroidies par des fluides différents de celui servant au refroidissement du cœur dans lequel elles sont installées, comme du sodium liquide) appelle naturellement l'utilisation de logiciels de simulation relativement sophistiqués en matière notamment de neutronique. La découverte en 2004 d'une fusion dans des crayons du cœur nourricier du réacteur CABRI (paragraphe 10.1.2), qui a eu pour origine une sous-estimation des températures atteintes dans les crayons concernés, confirme cette complexité.

\section{Neutronique}

- APOLLO : ce logiciel ${ }^{326}$ de simulation en deux dimensions (2D) dans le domaine de la neutronique, fondé sur la théorie du transport des neutrons (équation de Boltzmann), en état stable (stationnaire) mais pouvant simuler le « burn-up ${ }^{327}$ » du combustible (calcul dit d'évolution), pouvant prendre en compte un grand nombre de groupes d'énergie des neutrons (300 pour des calculs usuels), est principalement utilisé pour déterminer les « bibliothèques » de sections efficaces $^{328}$ pouvant ensuite être utilisées avec le logiciel CRONOS présenté ci-après. Il s'agit de « bibliothèques » multi paramétrées de sections efficaces (les paramètres pouvant être la température, la densité d'eau...), « condensées » en quelques groupes d'énergie et homogénéisées dans les "cellules » choisies pour la représentation du système étudié (un assemblage, un crayon, ou une plaque, une pastille...). Dans le principe ${ }^{329}$, APOLLO (2) permet aussi de déterminer les bilans neutroniques d'un cœur (production de neutrons par fission, absorptions et fuites) avec les paramètres neutroniques d'intérêt (bilans neutroniques tels que le facteur de multiplication effectif des neutrons $k_{\text {eff, }}$ paramètres cinétiques - temps de vie des neutrons, production de neutrons retardés -, contre-réactions neutroniques, efficacité des absorbants...).

- CRONOS : ce logiciel de simulation en trois dimensions de la neutronique d'un cœur de réacteur résout soit l'équation du transport soit l'équation de la diffusion, en utilisant la méthode des éléments finis à plusieurs groupes d'énergie des neutrons (deux groupes sont suffisants pour les calculs courants). Il permet de déterminer la distribution, en trois dimensions, de la puissance dans le cœur ainsi que les évolutions temporelles de cette puissance lors de transitoires incidentels ou accidentels, les efficacités des absorbants neutroniques... Le logiciel CRONOS peut également simuler le «burn-up » du combustible (calcul dit d'évolution). Les sections efficaces nécessaires au calcul proviennent de calculs réalisés avec le logiciel APOLLO et sont introduites comme données d'entrée. CRONOS est un code multi filière : rien dans son organisation ou sa structure ne préjuge du type de réacteur à calculer. De ce fait, des schémas de calcul utilisant CRONOS (2) ont été

326. Dans le domaine de la neutronique, les expressions « logiciel » et « schéma de calcul » sont à distinguer : un « schéma de calcul » désigne la séquence de modèles physiques associée à une « bibliothèque » bien définie de sections efficaces.

327. Consommation de combustible du fait de l'irradiation.

328. Les sections efficaces constituent des indicateurs de la probabilité d'interaction entre les neutrons et la matière, probabilité dépendant de l'énergie des neutrons.

329. Calcul très complexe avec la version 2 d'APOLLO; il sera plus aisé avec la version 3 . 
constitués (notamment en termes de maillage) pour de très nombreux réacteurs, incluant des réacteurs de recherche (figure 11.1).

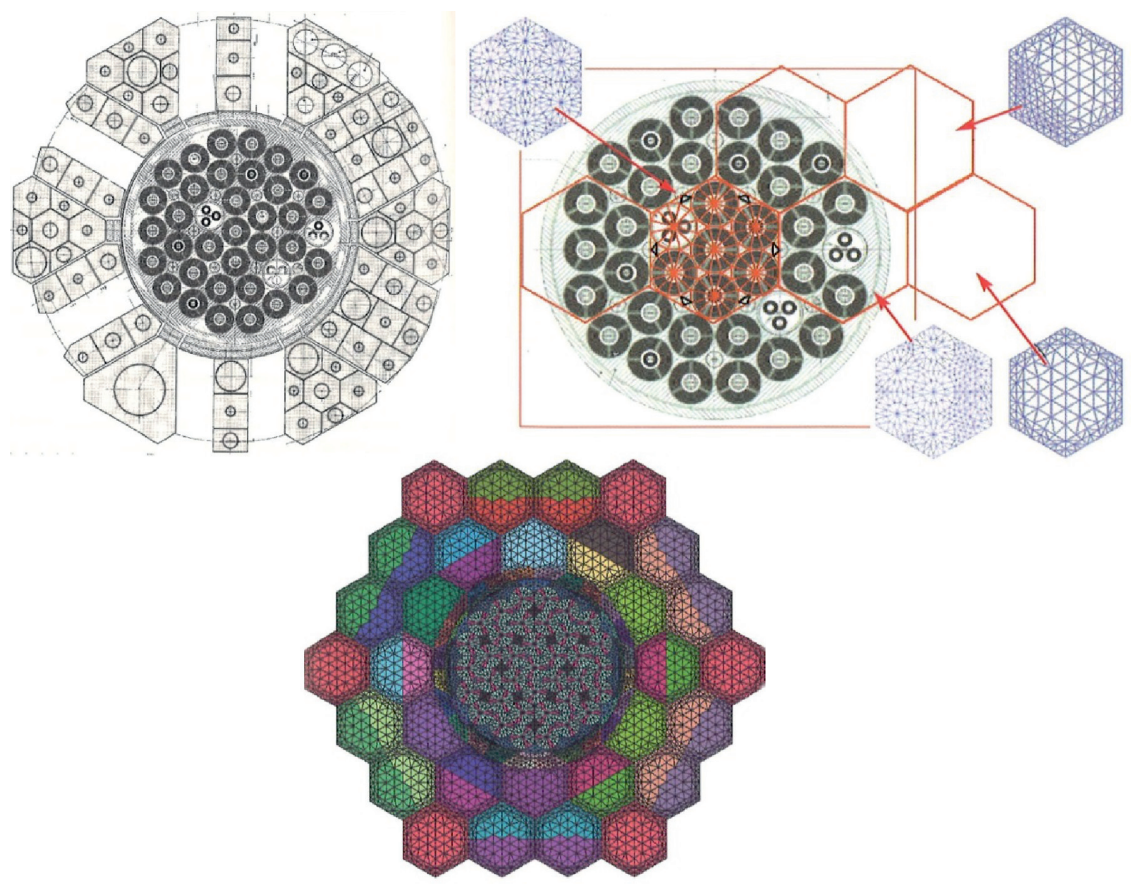

Figure 11.1. De la géométrie réelle du cœur du réacteur Jules Horowitz (en haut à gauche) au découpage en macro éléments hexagonaux (en haut à droite) et au maillage en éléments finis isoparamétriques (en bas) réalisé par le CEA pour le calcul, avec le logiciel CRONOS (2), de la distribution de puissance dans le cœur (source : monographie du CEA). @ DR.

- MCNP : ce logiciel de simulation en géométrie tridimensionnelle, développé par le Los Alamos National Laboratory, est historiquement le premier logiciel de simulation fondé sur la théorie du transport de particules et la méthode de Monte-Carlo (Monte Carlo N-Particule transport code). Le logiciel MCNP permet de traiter de nombreux types de particules (neutrons, électrons, photons...). Il est utilisé dans de nombreux domaines : outre la physique des réacteurs, peuvent être citées la radioprotection, la dosimétrie, la criticité ou encore la physique médicale.

Pour un cœur de réacteur, le principe du logiciel consiste à suivre l'histoire de chaque neutron dans le système étudié, de sa naissance (source externe, neutron de fission...) à sa mort (capture par un noyau ou fuite hors du système). Avec le logiciel MCNP, le choix est généralement fait d'utiliser un spectre continu d'énergie des neutrons, mais un spectre discrétisé peut aussi être utilisé. Si le logiciel MCNP peut simuler le « burn-up » du combustible (calcul dit d'évolution), il n'est pas apte (comme les autres logiciels de type Monte Carlo décrits ci-après, en l'état actuel de leur développement) à simuler des transitoires sur un réacteur, les contre-réactions neutroniques n'étant pas corrélées à la température. 
L'histoire de chaque neutron dépend de ses interactions avec la matière. La distance parcourue par le neutron entre deux collisions, les noyaux impliqués et les types d'interaction sont des paramètres échantillonnés aléatoirement en utilisant des résultats expérimentaux regroupés dans des "bibliothèques " de données nucléaires. Ainsi, en multipliant le suivi de nombreux neutrons, on peut simuler le comportement naturel du système et calculer des valeurs numériques approchées de certains paramètres neutroniques du cœur (bilans tels que le $k_{\text {eff }}$, coefficients de cinétique mais pas les contre-réactions dépendant de la température). Ce type de calcul reposant sur les probabilités, il est nécessaire de faire de nombreux tirages aléatoires pour réduire l'incertitude statistique ${ }^{330}$. Certains calculs peuvent durer plusieurs mois, d'où l'intérêt d'utiliser des calculateurs puissants. La représentation géométrique du système étudié repose sur une description géométrique précise de la surface d'objets, définis en fonction du problème à traiter et qui peuvent être de tailles très différentes (allant d'une zone d'un cœur à une pastille de combustible par exemple), représentation dite de type surfacique. Ainsi, le logiciel MCNP peut être utilisé pour des calculs précis de neutronique.

- TRIPOLI (TRIdimensionnel POLYcinétique) : ce logiciel de simulation en géométrie tridimensionnelle, développé par le CEA depuis les années 1960, résout par la méthode de Monte-Carlo l'équation du transport couplé des neutrons et des photons, ces derniers résultant des réactions nucléaires induites par les neutrons (fission ou capture - les photons se traduisent par le rayonnement $\gamma$ ). De la même manière qu'avec le logiciel MCNP, le choix est généralement fait avec TRIPOLI d'utiliser un spectre continu d'énergie des neutrons, mais un spectre discrétisé peut aussi être utilisé. Le logiciel TRIPOLI peut simuler le «burn-up »du combustible (calcul dit d'évolution), mais, pour la même raison que dans le cas de MCNP, il ne peut simuler des transitoires sur un réacteur. Avec TRIPOLI, le système étudié peut être traité par une définition de surfaces (comme dans le cas de MCNP) ou selon un mode combinatoire de volumes (l'utilisateur spécifiant alors le type de volumes et le lien entre les volumes). Ses domaines d'application privilégiés sont la physique des cœurs de réacteurs, la criticité et la radioprotection. Le logiciel TRIPOLI est très utilisé, en France, pour des calculs précis de neutronique (calculs dits étalons).

\section{Exemples d'utilisation}

Le CEA utilise le logiciel TRIPOLI pour des études de neutronique de ses réacteurs de recherche (réacteur CABRI, réacteur Jules Horowitz...). Il a également utilisé TRIPOLI (4) parallèlement avec APOLLO (2) ${ }^{331}$ pour examiner l'impact d'un combustible UMo sur la durée du cycle et sur les performances du réacteur à haut flux de l'Institut Laue-Langevin.

- MORET : ce logiciel de simulation, développé par l'IRSN depuis les années 1970, calcule le transport des neutrons par la méthode de Monte Carlo. Il est

330. L'incertitude statistique sur le résultat d'un calcul est donnée par le théorème de la limite centrale : l'écart-type sur le résultat est proportionnel à l'inverse de la racine carré du nombre de neutrons simulés.

331. Ouvrage du CEA cité au nota 322. 
généralement utilisé avec un spectre discrétisé en énergie des neutrons. La représentation géométrique est moins détaillée que ce qu'il est possible de faire avec des outils de maillage associés à MCNP et TRIPOLI. Le logiciel MORET permet, pour des systèmes complexes à trois dimensions contenant des matières fissiles, de déterminer les principales grandeurs suivantes (hormis les contreréactions corrélées à la température) : le facteur de multiplication effectif des neutrons $\left(k_{\text {eff }}\right)$, le flux neutronique, les taux de réaction (fission, absorption, diffusion) dans les différents volumes, les fuites de neutrons hors du système et les paramètres cinétiques du système (proportion de neutrons retardés et leurs temps de génération, durées de vie des neutrons...). La modélisation géométrique du système étudié est traitée selon le mode combinatoire de volumes. Le logiciel est plus particulièrement utilisé pour l'étude des risques de criticité dans les installations nucléaires (c'est-à-dire l'apparition d'une réaction en chaîne non maîtrisée en dehors des cœurs de réacteurs en fonctionnement), dans son « environnement » dénommé CRISTAL ${ }^{332}$, qui propose différents jeux de données (et d'autres logiciels tels que APOLLO (2) et TRIPOLI (4)).

\section{Exemples d'utilisation}

- Le logiciel MORET est principalement utilisé par l'IRSN pour ses expertises concernant les risques de criticité dans les installations du cycle du combustible. Mais, depuis une dizaine d'années, l'IRSN l'utilise aussi pour les réacteurs, comme ce fut le cas pour une étude destinée à tirer les enseignements d'une erreur de chargement de combustible survenue en 2001 dans le réacteur $n^{\circ} 4$ de la centrale nucléaire de production d'électricité située à Dampierre (département du Loiret). Au début des années 2010, l'IRSN a également utilisé MORET (5) pour simuler des essais réalisés dans les années 1960 dans le réacteur américain SPERT, qui visaient à étudier la réponse d'un cœur de réacteur à des insertions de réactivité par échelons successifs. Cette simulation a été menée dans le cadre d'une intercomparaison de logiciels de simulation organisée par l'AIEA (concernant les méthodes innovantes pour les réacteurs de recherche ${ }^{333}$ ), qui visait à apprécier l'aptitude de différents logiciels de simulation (utilisés dans le cadre de la conception de réacteurs, de leur démonstration de sûreté ou de l'expertise de cette démonstration) à reproduire un certain nombre de mesures faites directement sur différents cœurs de réacteurs de recherche, de natures neutronique et thermohydraulique. Le logiciel de simulation MORET (5) a notamment permis $^{334}$ de reproduire la répartition radiale de la puissance dans les

332. Le formulaire CRISTAL est développé et qualifié dans le cadre d'une collaboration entre l'IRSN, le CEA, AREVA-NC (Orano) et AREVA-NP (Framatome). Cet ensemble comprend des « bibliothèques » de données nucléaires, des procédures de calcul, des logiciels de simulation et des outils d'interface. Sa vocation est d'évaluer les conditions de criticité des installations nucléaires et des emballages de transport de matières fissiles.

333. IAEA Coordinated Research Project 1496 (2008-2013) : Innovative Methods in Research Reactor Analysis. Publication finale en préparation.

334. Voir la communication de l'IRSN à la conférence TOPSAFE 2012 Interpretation of reactivity accident transient on research reactors on example of SPERT-IV-D 12/25 Benchmark, Ivanov E., Maas L., Écrabet F. 

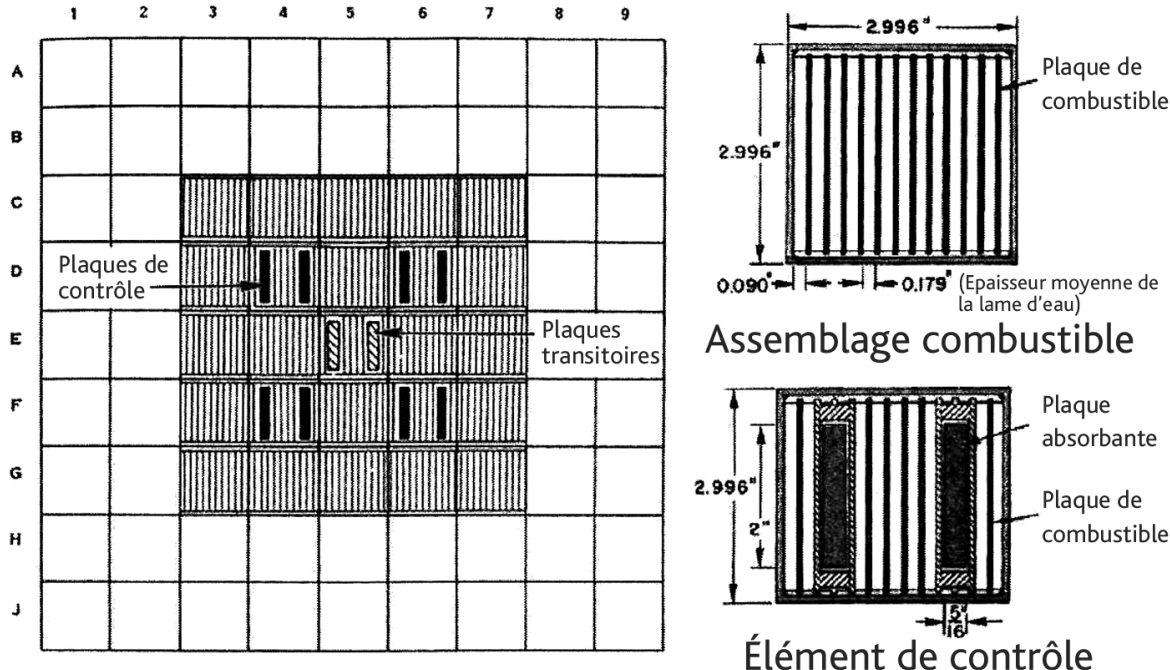

Figure 11.2. À gauche, schéma global du cœur SPERT-IV-D 12/25, à droite un élément combustible et un élément de contrôle contenant des plaques absorbantes. () Phillips Petroleum Company-Atomic Energy Division.

assemblages du cœur SPERT-IV-D 12/25 (voir la figure 11.2), qui a été utilisée ensuite pour des calculs d'échauffement de ces assemblages avec le logiciel CESAR du logiciel ASTEC (voir plus loin).

- En 2008, dans le cadre de la réévaluation de l'accident de type BORAX pour le réacteur ORPHEE, le CEA avait considéré que les insertions de réactivité enveloppes qu'il estimait envisageables pour ce réacteur n'étaient pas de nature à conduire à une interaction à caractère explosif de combustible fondu avec l'eau (explosion de vapeur). Dans le but d'apprécier le bien-fondé de cette conclusion, l'IRSN a réalisé en 2010 une étude avec le logiciel MORET (5) pour déterminer indépendamment les insertions de réactivité pour les scénarios retenus par le CEA, dont celui de rupture simultanée des deux « sources froides », de la « source chaude » et des neuf doigts de gant horizontaux (figure 11.3).

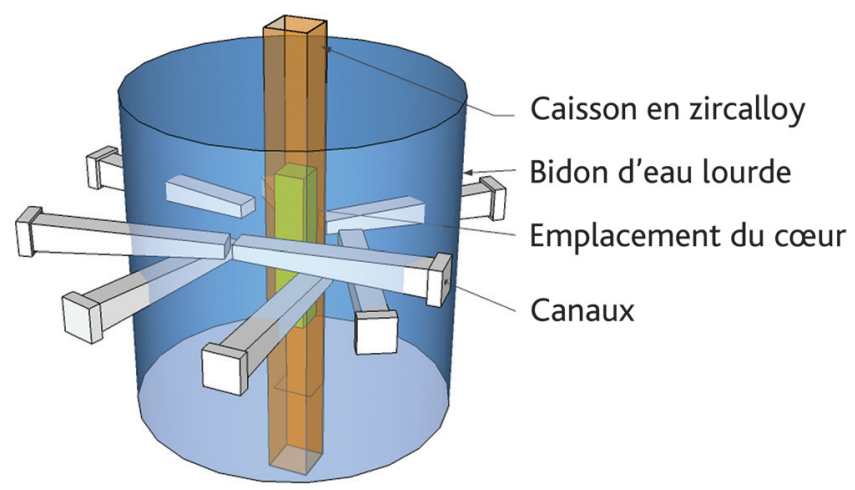

Figure 11.3. Modélisation en 3D du réacteur ORPHEE, notamment des canaux neutroniques, réalisée préalablement à la mise en œuvre du logiciel de simulation MORET. @ IRSN. 
Physiquement, ces équipements, dont la plupart contiennent un gaz, créent des espaces de fuite de neutrons, qui ne participent donc plus à la réaction en chaîne. Si de l'eau lourde vient envahir ces espaces, l'effet de réflexion des neutrons par l'eau lourde est accru, ce qui a pour effet d'augmenter la réactivité du cœur. La modélisation du réacteur utilisée a été affinée de façon à retrouver un certain nombre de paramètres neutroniques issus des calculs réalisés au moment de la conception du réacteur (avant 1980) - avec les logiciels TRIPOLI et TRIDENT - ou mesurés, comme le coefficient multiplicatif $k_{\text {eff, }}$ pour différentes positions des absorbants, la cote critique des absorbants, leur efficacité en réactivité... L'étude de l'IRSN a conduit à une insertion de réactivité maximale significativement supérieure à celle issue des calculs réalisés par le CEA, ce qui a conduit ce dernier à actualiser ses propres études en utilisant une version plus récente du logiciel TRIPOLI, qui ont confirmé les résultats de l'IRSN. Il est alors apparu important pour l'IRSN de faire en sorte, notamment, que la défaillance simultanée de l'ensemble des doigts de gant horizontaux puisse être écartée, en assurant une ductilité suffisante du matériau (alliage AG3NET) des doigts de gant en fin de vie. Une sous-estimation de la fluence ${ }^{335}$ reçue par ces doigts de gant conduisit l'exploitant à revoir son calendrier de remplacement des doigts de gant. L'Autorité de sûreté nucléaire demanda plus précisément (sur l'avis du GPR) que l'exploitant vérifie que le calendrier de remplacement des doigts de gant horizontaux et des chaussettes des " sources froides " permette de garantir que le " poids » en réactivité des dispositifs présentant simultanément une ductilité « très faible » reste limité (l'exploitant devant définir précisément les critères de ductilité et de « poids » en réactivité considérés).

- Une étude similaire ${ }^{336}$ a été réalisée en 2011 par l'IRSN avec le logiciel MORET (5) pour évaluer le caractère enveloppe de l'insertion de réactivité retenue par le CEA dans son étude de l'accident de type BORAX pour le réacteur Jules Horowitz. L'insertion de réactivité correspond à l'éjection d'une barre de contrôle contenant du hafnium, constituant le matériau absorbant des neutrons. Une valeur enveloppe de l'insertion de réactivité avait été déterminée par le CEA sur la base de calculs réalisés avec les logiciels APOLLO (2), CRONOS (2), TRIPOLI (4). L'objectif de l'étude de l'IRSN était de vérifier ce caractère enveloppe, notamment par des calculs de sensibilité à différents paramètres. Le logiciel MORET (5) a permis de déterminer l'insertion de réactivité par la différence de deux valeurs du coefficient de multiplication effectif $\left(k_{\text {eff }}\right)$ calculées pour deux états du cœur : barre de contrôle insérée et barre de contrôle éjectée (laissant place à un « trou d'eau »).

335. Grandeur sur laquelle est établie la durée de vie maximale pour les doigts de gant (voir le nota 255).

336. Pour cette étude et la précédente, voir la publication de l'IRSN présentée dans le cadre d'une conférence organisée par l'AIEA à Rabat au Maroc en 2011, intitulée Safety approach of BORAX type accidents in French research reactors, Chegrani Y., Gupta F., Tiberi V., Heulers L. 
Une vérification préalable ${ }^{337}$ de la modélisation utilisée avec le logiciel MORET (5) a été effectuée sur une configuration du cœur, en comparant certains

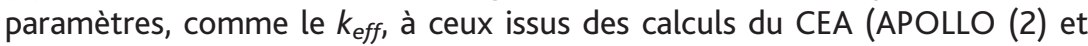
TRIPOLI (4)).

Les paramètres étudiés dans les calculs de sensibilité ont été le taux de combustion du combustible du cœur, la configuration initiale des barres de contrôle, la réactivité des dispositifs expérimentaux (figure 11.4).

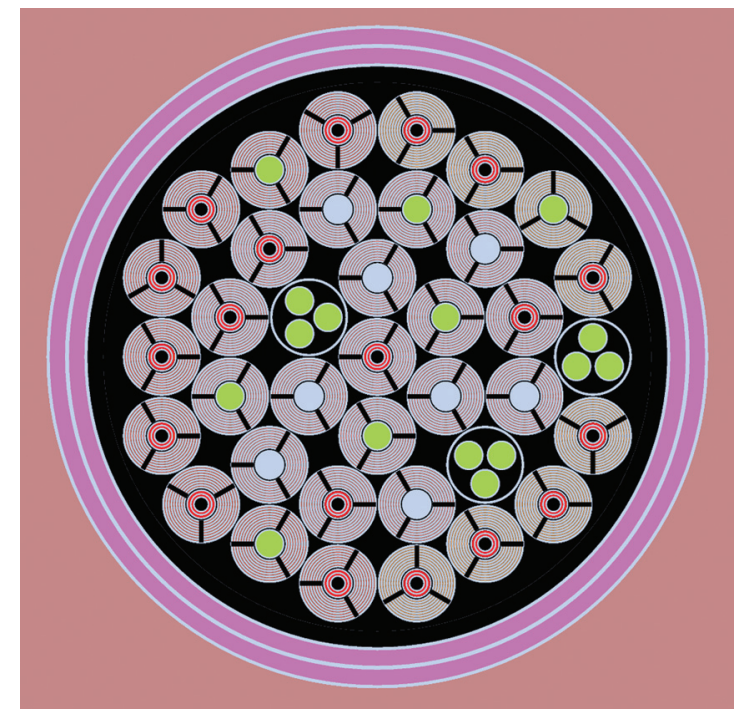

Figure 11.4. L'une des configurations du cœur du réacteur Jules Horowitz étudiée par l'IRSN avec le logiciel MORET (5) (en rouge les barres insérées, en bleu les barres extraites, en vert les dispositifs expérimentaux). ( ) IRSN.

Les résultats obtenus avec le logiciel MORET (5) ont confirmé les résultats des études du CEA concernant l'insertion de réactivité en cas d'éjection d'une barre de contrôle, notamment : le « poids » en réactivité plus important des barres absorbantes dans les assemblages de $1^{\text {ère }}$ couronne du cœur, le caractère pénalisant du cœur neuf par rapport à un cœur irradié. Ils ont aussi montré la faible influence de la réactivité des dispositifs expérimentaux sur l'insertion accidentelle de réactivité.

\section{Thermohydraulique}

- CATHARE (Code avancé de thermohydraulique pour les accidents de réacteurs à eau) : ce « code système ${ }^{338}$ » de thermohydraulique diphasique est développé et

337. L'accord a été trouvé en utilisant la même bibliothèque de sections efficaces que celle utilisée par le CEA.

338. Un « code système » permet la modélisation de l'ensemble d'un circuit et de ses composants (combustibles, échangeurs, pompes, structures...). 
utilisé principalement pour des études de sûreté des réacteurs à eau sous pression (étude du comportement thermohydraulique des réacteurs lors de transitoires incidentels ou accidentels, mise au point des procédures associées) et pour des travaux de recherche et développement. Il est aussi intégré dans le simulateur SOFIA 339 de l'IRSN.

Le logiciel CATHARE est développé conjointement par le CEA, EDF, AREVA-NP et l'IRSN, depuis 1979. La modélisation du cœur et des circuits retenus pour une étude peut être monodimensionnelle (1D), avec un cœur représenté par un canal ou assemblage « moyen »; mais le logiciel CATHARE possède également un module 3D permettant une représentation tridimensionnelle de la cuve et du cœur.

\section{Exemple d'utilisations}

Au début des années 2010, le CEA, pour l'établissement du rapport préliminaire de sûreté du réacteur Jules Horowitz, ainsi que l'IRSN pour l'expertise de ce rapport, ont utilisé le logiciel CATHARE (2) pour étudier l'accident de « rupture guillotine de l'élément particulier » (RGEP) de ce réacteur (collecteur, unique, d'alimentation en eau du cœur - voir la figure 5.11). L'objectif visé était de s'assurer que ce type de rupture ne pouvait pas constituer un initiateur d'une fusion du cœur du réacteur. Les critères retenus à cette fin étaient un taux de vide nul dans le cœur (pas d'ébullition) et une température maximale des gaines des plaques combustibles de $400{ }^{\circ} \mathrm{C}$ (afin d'éviter une rupture par fluage).

Les deux cas étudiés correspondent (figure 11.5) à une rupture guillotine doublement débattue dans la piscine et à une rupture guillotine à débattement limité dans un local (casemate, la tuyauterie disposant, dans cette casemate, d'un dispositif anti-débattement). Plusieurs conditions ont été retenues pour l'état initial du réacteur juste avant la rupture, notamment celles qui apparaissaient $a$ priori les plus pénalisantes (puissance maximale du réacteur, débit minimal de refroidissement du cœur, pression minimale de l'eau à la sortie du cœur, température minimale de l'eau à l'entrée du cœur, niveau minimal de l'eau dans la piscine du réacteur). Les simulations réalisées ont notamment montré :

- un déclenchement automatique quasi immédiat de l'arrêt d'urgence par franchissement du seuil de pression basse à la sortie du cœur ;

- l'établissement d'un écoulement gravitaire, en provenance de la piscine, dans les lignes d'aspiration de sauvegarde, qui vient compenser le débit sortant par la brèche et permet de conserver un inventaire en eau satisfaisant dans le circuit primaire principal ;

339. SOFIA (Simulateur d'observation du fonctionnement incidentel et accidentel) est un système informatique utilisé par l'IRSN pour des études et pour la formation. Il permet le calcul et le suivi en temps réel de l'évolution des paramètres physiques d'un réacteur nucléaire de type à eau sous pression. Il permet de simuler des défaillances de matériels et les actions des opérateurs. Le calcul peut être arrêté à un instant donné pour examiner l'état de l'installation et il est possible de revenir en arrière pour modifier le scénario étudié. Les réacteurs modélisés dans SOFIA sont ceux du parc électronucléaire français (réacteurs de 900 MWe, 1300 MWe, 1450 MWe et EPR). 


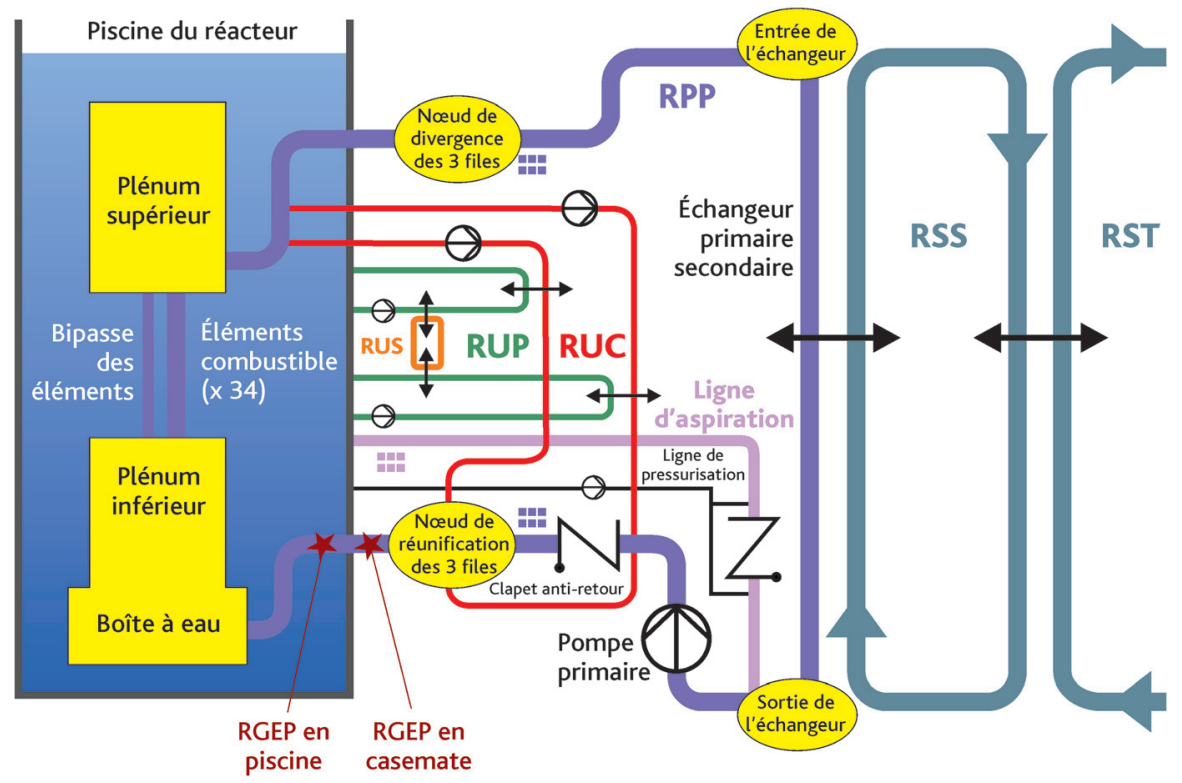

Figure 11.5. Schéma des circuits du réacteur Jules Horowitz et position des ruptures guillotines (RGEP) étudiées. (c) Georges Goué/IRSN.

- pour la rupture dans la casemate, une diminution du débit sortant par la brèche au fur et à mesure que cette casemate se remplit d'eau et que la partie rompue de la tuyauterie est noyée.

Les marges minimales par rapport aux critères retenus sont généralement atteintes juste après l'arrêt d'urgence.

L'étude menée par l'IRSN a notamment permis d'évaluer la sensibilité des résultats obtenus par le CEA - montrant le respect des critères indiqués plus haut - à certaines hypothèses, concernant par exemple le comportement des pompes primaires (risque de cavitation) juste après la survenue d'une brèche, ou encore le temps d'ouverture de la brèche. Cette étude a permis d'identifier un risque de non-respect des critères, et dès lors qu'il était nécessaire que le CEA apporte des éléments de nature à justifier que, même si les pompes primaires fonctionnaient (de façon temporaire) en mode dégradé, un débit suffisant d'eau traverserait encore les pompes pour refroidir le cœur.

Par ailleurs, le logiciel CATHARE (2) a permis au CEA de déterminer les efforts subis notamment par le dispositif anti-débattement lors d'une rupture dans la casemate, efforts dont la connaissance était nécessaire pour le dimensionnement mécanique de ce dispositif. Les calculs du CEA ont montré toute l'importance de ce dispositif, une rupture doublement débattue en casemate pouvant conduire à une fusion du cœur.

- FLICA, DULCINEE : ces logiciels permettent de simuler la thermohydraulique dans un cœur de réacteur et la thermique du combustible. Ils sont utilisés depuis 
plusieurs décennies pour les réacteurs de recherche français. Le logiciel DULCINEE dispose d'un modèle de neutronique dit de « cinétique point » (ou « OD »), qui permet de réaliser des calculs couplant une neutronique simplifiée et la thermohydraulique.

Le logiciel FLICA (4) permet une représentation tridimensionnelle d'un cœur de réacteur et traite les deux phases du fluide de refroidissement (liquide et vapeur). Pour les transferts thermiques dans le combustible, la modélisation est monodimensionnelle (1D).

En association avec le logiciel CRONOS, le logiciel FLICA peut être utilisé pour une représentation plus fine (3D) du cœur pour les études de transitoires menées avec le « code système » CATHARE. La figure 11.6 représente le couplage disponible dans la chaîne HEMERA.

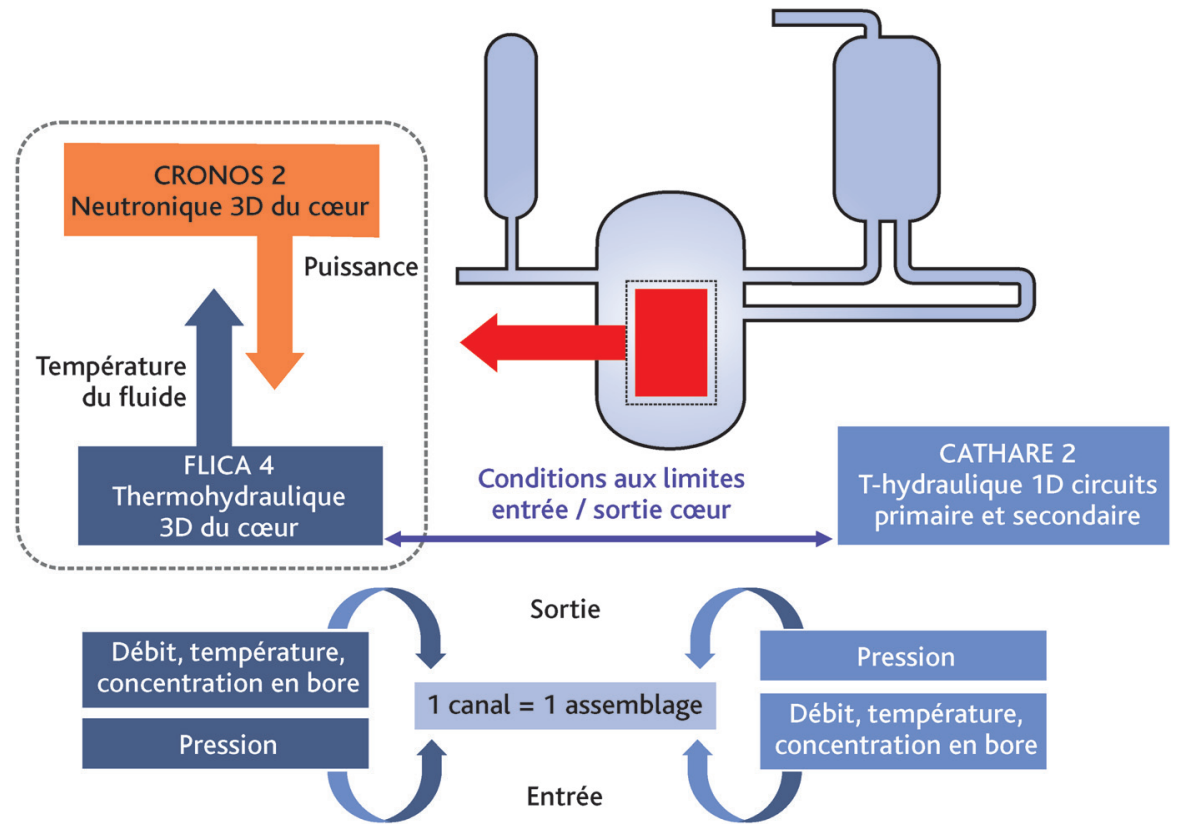

Figure 11.6. Couplage des logiciels CRONOS (2), FLICA (4) et CATHARE (2) (chaîne HEMERA) : paramètres d'interface entre ces trois logiciels. ( ) IRSN.

\section{Exemple d'utilisations}

Pour déterminer l'énergie thermique déposée dans le combustible du réacteur Jules Horowitz dans le cas de l'insertion accidentelle de réactivité retenue lors de l'étude de l'accident de type BORAX (éjection d'une barre de contrôle), le CEA a mis en œuvre ${ }^{340}$ un couplage des logiciels CRONOS (2) et FLICA (4) (sans

340. Voir notamment la communication du CEA au congrès TOPSAFE 2008 : The BORAX accident in the JHR, Maugard B., Elie J.-P., Trémodeux P., Iracane D., Lemoine P., Ratel G., Berthoud G. et al. 
modélisation de la dilatation des plaques combustibles, phénomène qui réduit l'épaisseur des canaux d'eau entre les plaques et donc apporte une antiréactivité). Il a également utilisé le code de « cinétique point » DULCINEE pour des études de sensibilité, ce logiciel («OD ») étant adapté à un petit cœur tel que celui du réacteur Jules Horowitz.

- Codes CFD (Computational Fluid Dynamics) : l'utilisation de ce type de logiciels de simulation est croissante, y compris pour les réacteurs de recherche, pour déterminer les écoulements de fluide à l'échelle locale par résolution des équations de Navier-Stokes moyennées dans le temps et dans l'espace, sur un domaine discrétisé par des mailles de dimensions allant du millimètre au centimètre.

\section{Exemples d'utilisations}

- En 2010, l'Institut Laue-Langevin a réalisé, en collaboration avec le laboratoire national d'Argonne (ANL, Illinois, États-Unis), des études ${ }^{341}$ de faisabilité d'une « conversion » du RHF à du combustible à faible enrichissement en uranium 235, de type UMo. Deux logiciels de type CFD ont été utilisés : le logiciel STAR-CD (utilisé par l'ANL) et le logiciel CFX développé par ANSYS ${ }^{342}$ (utilisé par l'ILL). La validité des modélisations a été vérifiée par des comparaisons à des mesures faites en réacteur et par des intercomparaisons des résultats de différents modèles. Ces études ont montré dans une première étape que le changement de combustible sans aucune modification des plaques de combustible conduirait à une dégradation notable des performances du réacteur, en termes notamment de flux neutronique. D'autres conceptions de l'élément combustible ont été étudiées. L'une d'elles, qui se traduirait par une augmentation de la quantité de combustible sans modification des dimensions externes des plaques, permettrait de conserver de bonnes performances du réacteur tout en procurant des marges de sûreté par rapport au risque d'ébullition dans les canaux d'eau situés entre les plaques. La mise en œuvre d'une « conversion » du cœur du RHF demeure toutefois soumise à la mise au point et à la qualification d'un nouveau combustible à plus forte densité que l'UAl.

- En 2010, l'Institut Laue-Langevin a également utilisé un code CFD (CFX) pour étudier le comportement des doigts de gant du RHF, pour montrer l'absence de fusion de l'élément combustible du cœur dans le cas de l'insertion de réactivité qui résulterait de la rupture d'un ou de plusieurs doigts de gant.

341. Voir notamment la communication ANL-ILL au congrès RERTR 2010 consacré à la réduction de l'enrichissement des combustibles pour les réacteurs de recherche et d'essais : Thermal-hydraulic safety analyses for conversion of the Laue Langevin Institute (ILL) High Flux Reactor (RHF) from HEU to LEU fuel, Tentner A., Thomas F., Bergeron A., Stevens J. (http://www.rertr.anl.gov/RERTR32/ pdf/S10-P4_Tentner.pdf).

342. ANSYS Inc. est une société américaine. 


\section{Thermomécanique}

- SCANAIR : ce logiciel, développé par l'IRSN depuis 1990, permet tout particulièrement de simuler le comportement thermomécanique des crayons de combustible des réacteurs à eau sous pression au cours de transitoires de puissance et d'évaluer les risques associés de perte d'étanchéité ou de rupture des gaines. Il est notamment utilisé pour la définition, la préparation et l'interprétation d'essais de tenue de crayons de combustible lors de tels transitoires, tels que ceux qui ont été ou seront réalisés dans le cadre du programme CIP dans le réacteur CABRI. Le logiciel SCANAIR permet de simuler des insertions rapides de réactivité (Reactivity Injection Accidents [RIA]) ou des rampes lentes de puissance telles que celles qui pourraient résulter d'une rupture de tuyauterie de vapeur ou encore d'un retrait incontrôlé d'une grappe d'éléments absorbants dans un cœur de réacteur à eau sous pression. Le logiciel SCANAIR modélise notamment les interactions thermomécaniques entre les pastilles de combustible $\left(\mathrm{UO}_{2}, \mathrm{UPuO}_{2}\right)$ et les gaines des crayons, l'ébullition du fluide réfrigérant (eau) et les différents mécanismes de déformation des gaines.

\section{Exemple d'utilisations}

Dans sa recherche de l'explication de la fusion de crayons de combustible du cœur nourricier du réacteur CABRI découverte en 2004 (paragraphe 10.1.2), le CEA, exploitant de ce réacteur, a mis en œuvre plusieurs logiciels de simulation, parmi lesquels peuvent être cités APOLLO (2), TRIPOLI (4), DULCINEE et SCANAIR. Comme cela a été indiqué au paragraphe 10.1.2, le CEA en a conclu que les effets des transitoires réalisés dans CABRI sur les crayons du cœur nourricier étaient mal estimés dans les études de sûreté conduites préalablement à la réalisation de ces transitoires. Le CEA a alors décidé de mettre au point un nouvel outil de calcul pour la réalisation des études préalables aux futurs essais du programme CIP dans la boucle à eau sous pression. Cet outil associe le logiciel SCANAIR ${ }^{343}$ à des jeux de données appropriés. Dans le cadre de l'expertise du dossier transmis par le CEA visant à démontrer que le cœur nourricier pourrait subir sans dommage les essais du futur programme expérimental CIP, l'IRSN, étant le développeur du logiciel SCANAIR, a fait réaliser l'expertise du nouvel outil du CEA par la société belge AVN, qui n'a pas émis de contre-indication à l'utilisation de cet outil. De plus, l'IRSN a utilisé le logiciel SCANAIR pour une étude ${ }^{344}$ visant à apprécier la validité des nouveaux critères de tenue des gaines des crayons du cœur de CABRI proposés par le CEA. L'objectif de cette étude de l'IRSN était d'évaluer la cohérence de ces nouveaux critères avec les résultats d'un certain nombre d'essais réalisés dans les

343. Le CEA avait dans un premier temps envisagé un chaînage des logiciels de simulation CATHARE et SCANAIR, mais il a décidé en définitive d'utiliser le logiciel SCANAIR seul en réalisant un important travail de calibration et de validation du module thermohydraulique pour l'adapter à la configuration du cœur nourricier de CABRI.

344. Voir la communication faite par l'IRSN à la conférence IGORR 12 en 2009 : Analysis of CABRI driver core new safety demonstration for fuel rods integrity during fast power transients, Écrabet F., Pelissou C., Moal A. 
réacteurs SPERT aux États-Unis et NSRR au Japon. Ces essais avaient permis de déterminer un seuil de rupture exprimé en termes d'énergie déposée dans le combustible pour les gaines en acier inoxydable (environ $240 \mathrm{cal} / \mathrm{g}$ ). Pour mener cette étude, il était indispensable d'utiliser strictement la même version du logiciel SCANAIR et ses jeux de données que ceux mis au point par le CEA en vue de ses propres calculs de l'impact des futurs essais CIP sur le cœur nourricier. Le CEA a mis à la disposition de l'IRSN ces éléments. L'étude de l'IRSN a alors montré que les nouveaux critères, exprimés ${ }^{345}$ en termes de température maximale des gaines $\left(1300^{\circ} \mathrm{C}\right)$ et de déformation équivalente maximale des gaines $(3,65 \%)$, étaient cohérents avec un seuil de rupture de $240 \mathrm{cal} / \mathrm{g}$.

\section{- Accidents de fusion de combustible}

- SIMMER : ce logiciel, où la neutronique et la mécanique des fluides sont couplées, permet de simuler un accident de fusion de combustible dans un réacteur à neutrons rapides. Il a été développé initialement à Los Alamos, à partir de 1974. Les organismes PNC (Power reactor and Nuclear fuel development Corporation, Japon), FzK (Forschungszentrum Karlsruhe, Allemagne, devenu Karlsruher Instituts für Technologie [KIT]) et le CEA poursuivent son développement pour des études sur les réacteurs à neutrons rapides de quatrième génération. En France, il a été utilisé dans les années 1980 et 1990 pour l'étude des accidents hypothétiques de fusion du cœur dans les réacteurs à neutrons rapides (principalement SUPERPHENIX).

\section{Exemple d'utilisations}

Dans les années 2000, l'IRSN a, avec FzK, adapté le logiciel SIMMER-III au réacteur Jules Horowitz, pour des études à caractère exploratoire d'un accident de type BORAX. De nombreuses adaptations ont été nécessaires, notamment pour pouvoir simuler correctement le comportement neutronique du cœur dans le domaine des neutrons thermiques ainsi que les combustibles de ces réacteurs sous forme de plaques cintrées ${ }^{346}$... Ces études ont notamment montré que l'énergie déposée dans un cœur tel que celui du réacteur Jules Horowitz pourrait éventuellement dépasser la valeur forfaire de $135 \mathrm{MJ}$, et que dès lors les séquences en cause (par exemple l'éjection simultanée de plusieurs barres absorbantes) devraient être rendues très improbables (par des dispositions robustes de conception, fabrication et surveillance en service).

345. Outre l'absence de fusion de combustible (la température de fusion de l' $\mathrm{VO}_{2}$ est d'environ $2840^{\circ} \mathrm{C}$ ).

346. Communications de l'IRSN : International Conference on the Physics of Reactors PHYSOR 2008 Upgrading of the coupled neutronics-fluid dynamics code SIMMER to simulate the research reactors core disruptive RIA, Biaut G. et al. ; congrès TOPSAFE 2008 Reevaluation of BDBA consequences of research reactors, Biaut G. et al. Voir aussi la communication commune IRSN-CEA faite à la $18^{\mathrm{e}}$ International conference on Nuclear Engineering (ICONE) en 2010 : Validation of SIMMER III neutronics module for the simulation of reactivity injection accident in material testing reactors, Chegrani Y., Ivanov E., Di Salvo J., d'Aletto C. 
- MC3D : MC3D est un logiciel de thermohydraulique multiphasique développé par le CEA puis par l'IRSN, qui permet de simuler l'explosion de vapeur qui résulterait d'une interaction thermodynamique entre du combustible (notamment lorsqu'il est à l'état liquide) et le réfrigérant d'un réacteur ; un tel phénomène pourrait survenir au cours d'un accident de fusion d'un cœur de réacteur. Ce logiciel permet notamment de déterminer les pressions dynamiques exercées sur des structures (par exemple les parois d'une piscine de réacteur). Il simule d'abord une première phase de l'interaction thermodynamique, appelée prémélange, consistant en un mélange grossier des deux liquides, accompagnée d'une vaporisation plus ou moins forte. Dans certaines conditions, le prémélange peut être déstabilisé, ce qui peut conduire à une explosion violente s'apparentant à une détonation (seconde phase).

\section{Exemple d'utilisation}

Le CEA a utilisé le logiciel MC3D dans l'étude des interactions entre le combustible fondu et l'eau lors d'un accident de type BORAX pour le réacteur Jules Horowitz ${ }^{347}$, notamment pour déterminer les sollicitations que pourraient subir la cuve et les raccordements des tuyauteries du bloc-pile dans un premier temps, les parois et le fond de la piscine du réacteur dans un second temps; ces sollicitations résulteraient des ondes de choc et de leurs réflexions multiples, ainsi que de la détente de la bulle de vapeur d'eau.

- ASTEC : le système de logiciels de simulation ASTEC (Accident Source Term Evaluation Code) a pour ambition de simuler l'ensemble des phénomènes qui interviendraient au cours d'un accident de fusion du cœur d'un réacteur à eau, depuis l'événement initiateur jusqu'aux éventuels rejets de produits radioactifs à l'extérieur de l'enceinte de confinement, hormis l'explosion de vapeur qui peut être traitée avec le logiciel MC3D et les sollicitations subies par les structures qui peuvent être traitées avec un logiciel tel que Cast3M (voir plus loin). Le logiciel ASTEC (voir la figure 11.7) a été développé en commun depuis de nombreuses années par l'IPSN puis l'IRSN avec son homologue allemand la GRS ; depuis, l'IRSN en poursuit seul le développement. Les applications d'ASTEC concernent principalement l'analyse de sûreté des réacteurs à eau sous pression avec l'évaluation des rejets radioactifs pouvant résulter de la fusion du cœur d'un tel réacteur et l'examen des procédures à mettre en œuvre en cas de survenue d'un tel accident. Le logiciel ASTEC est également utilisé par l'IRSN pour ses études probabilistes de sûreté de niveau 2 relatives aux réacteurs du parc électronucléaire. Enfin, il a été utilisé pour la préparation et l'interprétation des programmes expérimentaux, en particulier le programme d'essais intégraux Phébus-PF et les essais du programme ISTP (International Source Term Program ${ }^{348}$ ).

347. Communication du CEA citée au nota 340 .

348. Programme de recherche international sur le « terme source ». 


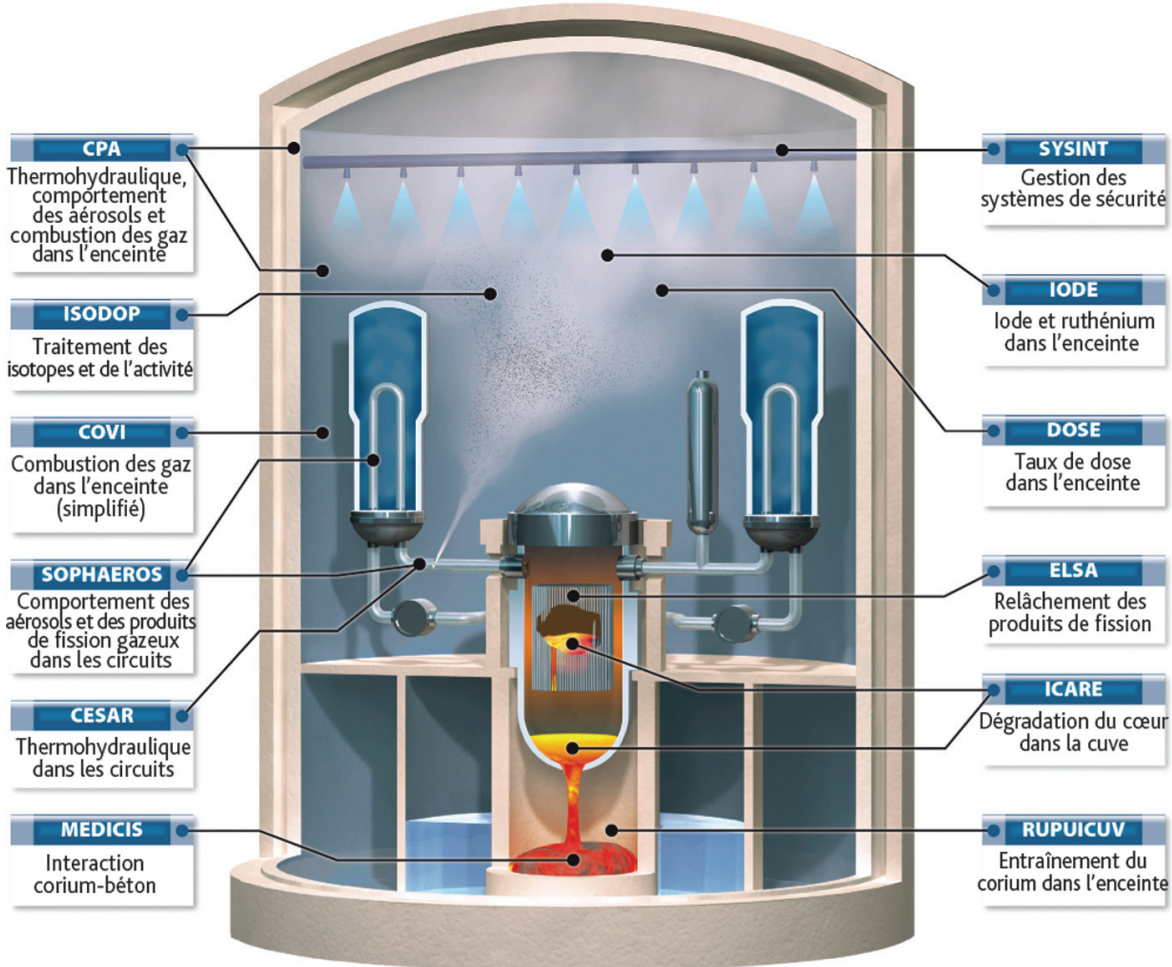

Figure 11.7. Les différents phénomènes intervenant lors d'un accident de fusion d'un cœur (réacteur à eau sous pression) et les modules les simulant dans le logiciel ASTEC. ( ) IRSN.

\section{Exemples d'utilisations}

- Le CEA a utilisé le logiciel IODE d'ASTEC pour étudier les transferts d'iode dans le bâtiment du réacteur du réacteur Jules Horowitz lors d'un accident de type BORAX - il a utilisé les logiciels CERES et GAZAXI pour l'évaluation des contributions des principaux radionucléides aux doses (effectives) au cours de leur migration dans l'environnement ${ }^{349}$.

- Dans le cadre de l'intercomparaison de logiciels de simulation organisée par l'AIEA évoqué précédemment (Coordinated Research Project concernant les « Innovative Methods in Research Reactor Analysis » - 2008-2013), l'IRSN a réalisé des calculs à l'aide du logiciel de thermohydraulique CESAR pour interpréter des essais d'insertion de réactivité réalisés dans le réacteur SPERT. Une adaptation du logiciel CESAR aux combustibles sous forme de plaques a été nécessaire. Il est apparu que ce logiciel permettait de retrouver les températures des gaines des plaques mesurées dans les

349. Communication du CEA citée au nota 340 . 
assemblages du cœur SPERT-IV-D 12/25 (voir précédemment pour la simulation de la neutronique avec le logiciel MORET (5)).

- L'IRSN a également utilisé le logiciel CPA dédié à la thermohydraulique dans l'enceinte de confinement pour évaluer l'efficacité d'une nouvelle gestion du confinement (de type dynamique et non plus statique) proposée par l'exploitant du réacteur à haut flux à Grenoble (RHF) pour les situations accidentelles $^{350}$. L'objectif était d'apprécier les conclusions tirées des calculs de l'exploitant visant à démontrer la possibilité d'un maintien du bâtiment du réacteur en dépression relative (par rapport à l'espace annulaire situé entre les deux enceintes) dans de telles situations, compte tenu de l'échauffement de l'air (dû aux produits de fission relâchés dans le bâtiment du réacteur et à l'échauffement de l'eau de la piscine dans le cas d'une fusion du combustible), des possibilités de fuites directes d'air vers l'environnement, du « gonflage » de l'espace annulaire situé entre les deux enceintes (enceinte interne en béton, enceinte externe métallique). Trois situations accidentelles ont été étudiées : un accident de type BORAX, une fusion de combustible sous eau, une fusion de combustible à l'air.

\section{Mécanique}

- Cast3M, ASTER, logiciels d'ANSYS : Cast3M est un logiciel de simulation par éléments finis dédié à la mécanique des structures et à la mécanique des fluides, développé par le CEA. ASTER (Analyses des structures et thermomécanique pour des études et des recherches) est un logiciel similaire, développé par EDF. ANSYS Inc. est une société américaine qui crée et diffuse différents logiciels de mécanique des structures (y compris pour des sollicitations conduisant à de grandes déformations).

Exemples d'utilisations :

- Cast3M est largement utilisé par les concepteurs et les exploitants pour les installations nucléaires françaises, pour des applications relatives à des structures métalliques ou à des ouvrages de génie civil (piscines et bâtiments de réacteurs...), notamment des réacteurs de recherche. Il est aussi largement utilisé par l'IRSN, qui peut en outre être amené à collaborer avec le CEA pour la réalisation de développements particuliers. Par exemple, dans le domaine du génie-civil, des développements ${ }^{351}$ consistent à mettre au point des lois pour simuler le comportement différé ou dynamique d'ouvrages en béton en cas de chargements accidentels (par

350. Voir la communication de l'IRSN faite à la conférence RRFM 2010 : Development of a numerical tool for safety assessment and emergency management of experimental reactors, Maas L., Beuter A., Seropian C.

351. Ces développements sont effectués dans le cadre de thèses, associant l'IRSN et d'autres partenaires, dont le CEA. 
exemple en cas de séisme...), qui sont ensuite intégrés dans Cast3M et ainsi mis à disposition de l'ensemble des utilisateurs de Cast3M.

- L'Institut Laue-Langevin a utilisé le logiciel ASTER pour les études de conception et de dimensionnement du nouveau bâtiment PCS 3 du RHF (faisant partie du « noyau dur » des dispositions post-Fukushima).

- EUROPLEXUS, LS-DYNA, RADIOSS : EUROPLEXUS est un logiciel de simulation par éléments finis de phénomènes de dynamique rapide, prenant en compte les structures et les fluides, développé à l'origine par le CEA (code PLEXUS) et le Centre commun de recherche (CCR) d'Ispra en Italie (PLEXUS-3C), puis repris par un groupe d'utilisateurs tels que EDF et l'ONERA. LS-DYNA est un logiciel de calcul du même type, développé aux États-Unis par la Livermore Software Technology Corporation (LSTC), de même que RADIOSS, développé par Altair Engineering. Ces logiciels permettent par exemple d'étudier le comportement de structures soumises à des chocs.

\section{Exemple d'utilisations}

Pour le réacteur Jules Horowitz, le CEA a utilisé ${ }^{352}$ les logiciels EUROPLEXUS et RADIOSS pour étudier le comportement des structures de la piscine du réacteur en cas d'accident de type BORAX - en modélisant une bulle de vapeur de caractéristiques telles qu'elle conduise aux surpressions préalablement déterminées avec le logiciel MC3D.

\section{- Évaluations en situations d'urgence}

En situations d'urgence ${ }^{353}$ ou lors des exercices de crise, les exploitants des réacteurs de recherche français ainsi que l'IRSN appuieraient ou appuient leurs appréciations sur des évaluations effectuées avec des logiciels de simulation plus ou moins simplifiés. En particulier, l'IRSN dispose d'un logiciel de simulation utilisé pour les installations autres que les réacteurs à eau sous pression du parc électronucléaire, qui permet de déterminer les transferts de produits radioactifs au sein d'une installation et les rejets dans l'environnement (quantité et cinétique de rejet pour chaque radionucléide). Ce logiciel modélise, de façon simplifiée, les fuites de radionucléides entre des locaux, les transferts par les systèmes de ventilation, ainsi que les rejets hors de l'installation. Les taux de dépôt (pour les aérosols) et l'efficacité des dispositifs de filtration y sont entrés comme des données. Ce logiciel est utilisé pour l'établissement et la mise à jour des fiches d'accidents-types (voir le paragraphe 7.7). Il sert également aux experts de l'IRSN à définir les scénarios joués lors des exercices de crise. Il peut éventuellement être aussi utilisé dans le cadre des expertises menées par l'IRSN. Un tel logiciel simplifié se prête bien à un pré-paramétrage avec les données pertinentes correspondant aux différents réacteurs de recherche, cela permettant de disposer de modèles utilisables rapidement en situations d'urgence ou lors des exercices.

352. Communication du CEA citée au nota 340.

353. En fait dès lors qu'un PUI est déclenché. 